\title{
Vers l'émergence d'un modèle du quartier commercial transnational ? Les quartiers du made in China au Caire et à Oran
}

The emergence of a transnational marketplace model: the "made in China" neighbourhoods of Cairo and Oran

Los barrios con mercados de productos chinos en El Cairo y Orán : ¿La

configuración distritos comerciales transnacionales?

\section{Anne Bouhali}

\section{OpenEdition}

\section{Journals}

Édition électronique

URL : http://journals.openedition.org/mappemonde/4076

DOI : 10.4000/mappemonde.4076

ISSN : 1769-7298

Éditeur

UMR ESPACE

Référence électronique

Anne Bouhali, « Vers l'émergence d'un modèle du quartier commercial transnational ? Les quartiers du made in China au Caire et à Oran », Mappemonde [En ligne], 128 | 2020, mis en ligne le 01 juillet 2020, consulté le 02 septembre 2020. URL : http://journals.openedition.org/mappemonde/4076 ; DOI : https://doi.org/10.4000/mappemonde.4076

Ce document a été généré automatiquement le 2 septembre 2020. 


\title{
Vers l'émergence d'un modèle du quartier commercial transnational? Les quartiers du made in China au Caire et à Oran
}

\author{
The emergence of a transnational marketplace model: the "made in China" \\ neighbourhoods of Cairo and Oran \\ Los barrios con mercados de productos chinos en El Cairo y Orán : ¿La \\ configuración distritos comerciales transnacionales?
}

Anne Bouhali

\section{Introduction}

1 Chaussures, jeans, vaisselle, tapis de prière, bijoux fantaisie et autres petits bibelots... Les produits de consommation courante en provenance de Chine, ou plus largement d'Asie, ont envahi les marchés et les rues des villes et des campagnes du Maghreb et du Machrek depuis la fin des années 1990 et l'ouverture de la région au libéralisme économique et à la mondialisation (Pliez, 2007 ; Choplin et Pliez, 2018). Leur succès est vif auprès des classes moyennes et populaires ayant atteint un petit niveau de prospérité (Darbon, 2012) et qui sont désireuses de consommer des produits jusqu'alors difficiles à trouver dans les marchés du sud de la Méditerranée. Depuis, on a assisté à la massification des importations de produits made in China dans la région, en lien avec l'augmentation de la demande des classes moyennes et populaires qui veulent avoir accès à des modes de consommation globalisés (Bouhali, 2018a).

2 Ces marchandises sont importées d'Asie et d'ailleurs (Dubaï, Istanbul) principalement par des commerçants maghrébins (Belguidoum et Pliez, 2012), et sont ensuite distribuées dans la région via des places marchandes qui jouent un rôle de concentration et d'éclatement des flux à toutes les échelles (Belguidoum et Pliez, 2015). 
Ces quartiers spécialisés dans l'importation sont parfois des centralités commerciales récentes, situées en dehors des grands centres urbains (Belguidoum et al., 2017). D'autres sont des marchés à l'histoire urbaine et commerciale plus longue, situés au cœur des grandes métropoles du monde arabe, et pour lesquels les flux transnationaux de marchandises représentent une nouvelle étape de développement urbain et commercial. À la massification des produits d'importation répond en effet un essor urbain et commercial de ces marchés populaires, ou souks ${ }^{1}$. C'est le cas des marchés du Muski, situé au cœur de la ville ancienne du Caire en Égypte, et de Médina J'dida à Oran, quartier péricentral de la deuxième ville d'Algérie, objets de cet article. Avec le développement du commerce transnational de biens de consommation courante à la fin du siècle dernier, ces deux quartiers sont devenus les portes d'entrée des marchandises made in China, à destination de toute l'Égypte pour l'un, de l'Ouest algérien pour l'autre, mêlant fonctions de vente en gros et au détail et entreposage.

Cet article s'appuie sur des enquêtes réalisées dans ces quartiers entre 2011 et 2014. Elles ont associé observation des quartiers à différents moments de l'année et de la journée, entretiens semi-directifs et discussions plus informelles avec des commerçants, du simple vendeur de rue au commerçant à la tête de plusieurs magasins, des propriétaires immobiliers et la clientèle qui fréquente ces marchés. Ces enquêtes nous ont permis de constater que la massification des flux de produits made in China, très visible dans les rues et sur les étals, bien que difficilement quantifiable ${ }^{2}$ (Mathews et Alba Vega, 2012), s'accompagne d'une uniformisation des paysages commerciaux ${ }^{3}$ de ces marchés, ainsi que d'une banalisation du tissu urbain. Cet article propose donc de montrer que, face au déploiement des routes de l'échange entre Asie, Maghreb et Machrek, on assiste à l'émergence d'un modèle de quartier de l'importation et à sa diffusion dans la région nord-africaine. À la mondialisation des échanges commerciaux et des mobilités de marchandises et de personnes répond ainsi une mondialisation des formes urbaines à l'intérieur des marchés qui distribuent les articles made in China. La mondialisation est ainsi un facteur-clé de la fabrique et de la requalification de la ville marchande, y compris dans les espaces discrets (Pliez, 2007; Choplin et Pliez, 2015) et « off the map » (Robinson, 2002), que sont parfois les villes des Suds.

4 En Égypte et en Algérie, les marchés du commerce transnational se recomposent sous l'effet de la mondialisation selon des processus et des caractéristiques communs. La construction d'un modèle de quartier commercial transnational se fait ainsi à plusieurs échelles, auxquelles apparaissent des caractéristiques communes qu'il s'agira de mettre en évidence. Ces trois échelles constitueront les trois moments de cet article. À l'échelle intra-urbaine, ces marchés mobilisés par les acteurs du made in China pour y développer leurs affaires sont des quartiers populaires péricentraux anciennement délaissés, situés à l'écart des quartiers touchés par la globalisation, comme les quartiers d'affaires ou les nouvelles centralités périphériques que l'on trouve au Caire (Abaza, 2006). Leur discrétion, leur caractère populaire et leur localisation à l'intérieur de la métropole sont ainsi des critères constitutifs de la centralité commerciale transnationale impulsée par le bas. À l'échelle de la rue commerçante ensuite, la construction du quartier commercial transnational se traduit par une uniformisation paysagère à travers l'envahissement des rues et des boutiques par les produits made in China à destination d'un marché régional de consommation et une réécriture du souk populaire. À l'échelle de l'̂llot enfin, le quartier du commerce transnational se reconnaît par une 
uniformisation du bâti qui résulte d'un intense renouvellement urbain, substituant au bâti hérité un bâti commercial standardisé.

\section{Des marchés populaires du made in China au cœur des métropoles cairote et oranaise}

5 Les quartiers dans lesquels le commerce transnational de produits fabriqués en Chine s'est ancré ne sont pas de simples supports aux circulations des marchandises et des commerçants ; ce sont leurs caractéristiques, à la fois économiques, urbaines et sociales qui sont à l'origine de leur ancrage dans deux quartiers commerçants a priori banals, des caractéristiques qu'on retrouve tant à Médina J'dida qu'au Muski.

6 Ces quartiers sont tout d'abord héritiers d'une histoire urbaine et commerciale longue, histoire qui a constitué un terreau social et spatial fertile pour le commerce transnational, ses acteurs et ses produits. Médina J'dida et le Muski sont deux quartiers anciennement en situation de relégation, et pour lesquels le développement récent du commerce transnational a permis une requalification. Médina J'dida est un quartier créé à l'instigation du colonisateur français à partir du milieu du XIXe siècle (Bekkouche, 2005). D'abord espace de relégation des populations arabes aux portes de la ville européenne largement issues des différentes vagues d'un important exode rural, la « Ville nouvelle » est progressivement devenue au $\mathrm{XX}^{\mathrm{e}}$ siècle un quartier péricentral d'Oran, puis un centre commerçant de plus en plus important à destination de la ville nouvellement indépendante et de sa région (Semmoud, 1975). Le Muski présente quant à lui une histoire plus longue. D'abord morceau de la ville médiévale cairote à vocation résidentielle, il s'est affirmé comme un quartier commercial dynamique à partir du XVIII ${ }^{e}$ siècle en lien avec l'appartenance de l'Égypte à l'Empire ottoman et au développement du grand commerce (textile, café, thé) à l'échelle de l'est de la Méditerranée. À la suite du déplacement du centre de gravité de la ville moderne au $\mathrm{XIX}^{\mathrm{e}}$ siècle dans le quartier de Wust al-Balad/Downtown, situé à l'ouest de la ville médiévale (figure 1), et d'une partie des activités du tertiaire supérieur (banques, assurances en particulier) autrefois localisées dans la ville ancienne (Abu-Lughod, 1965 ; Raymond, 1993), le quartier est pourtant resté à partir des années 1970 une centralité commerciale majeure et le cœur économique d'activités nombreuses: commerce de l'or, des épices, mais aussi «des produits manufacturés non sophistiqués » (Ilbert, 1982 ; Meyer, 1988), en même temps qu'il voyait se consolider un important tissu industriel. Médina J'dida à Oran et la ville ancienne du Caire, dans laquelle se trouve le Muski, sont donc tous deux des quartiers marchands qui se sont structurés sur le temps long. Les réseaux du commerce transnational constitués par des commerçants algériens et égyptiens se sont inscrits dans des espaces ayant déjà développé des relations économiques à des échelles régionales, voire même mondiales (Belguidoum et Pliez, 2012). L'essor des circulations de produits made in China et leur apparition dans les rues du Muski et de Médina J'dida ne représentent finalement qu'une nouvelle strate dans le développement urbain et commercial de ces quartiers.

7 Autre caractéristique constitutive des quartiers du made in China: aucun d'entre eux n'assure de fonctions centrales au sein de son agglomération (figure 1). Au Caire, l'hyper-centre accueillant les fonctions de commandement métropolitain est le centreville moderne, Wust al-Balad, qui concentre sièges sociaux de grandes entreprises mondiales et autres services du tertiaire supérieur. À Oran, cette fonction est assurée 
par le quartier al-Emir (avenue Larbi Ben M'hidi), dans laquelle on trouve notamment les boutiques de quelques grandes marques internationales de prêt-à-porter. Avec le Muski et Médina J'dida, on assiste au développement de centralités interstitielles, « quotidiennes, banales (...) impulsées par le bas » (Bertoncello et Fettah, 2007), qui ont justement pu se développer parce qu'elles se trouvent à côté (à l'ombre ?) des espaces centraux du pouvoir et de la mondialisation « par le haut ».

Figure 1. Médina J'dida et le Muski, deux centralités populaires anciennes, situées dans des quartiers péricentraux des métropoles oranaise et cairote

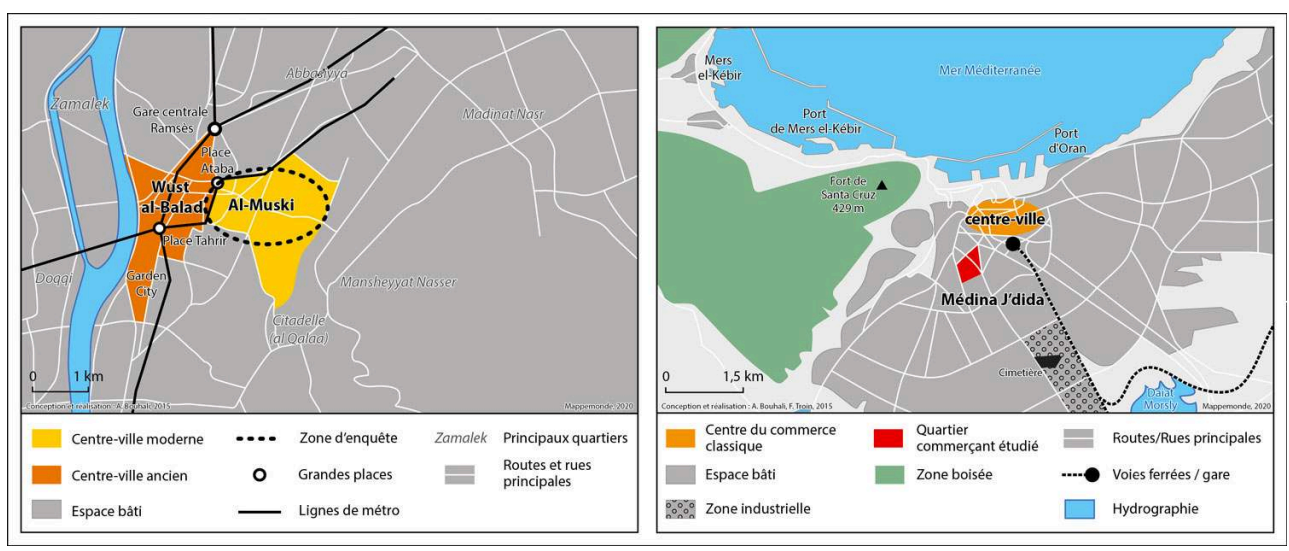

Ces places marchandes sont ainsi des centralités commerciales bien connues et fréquentées par les classes moyennes et populaires urbaines, centralités qui bénéficient d'ailleurs d'une réputation qui dépasse les limites de ces villes. Médina J'dida est une place marchande d'échelle régionale, relais pour tout l'Ouest algérien des flux de marchandises made in China qui entrent dans le pays par les grandes places marchandes de l'Est ou par Alger (Belguidoum et Pliez, 2012 ; Bouhali, 2015), tandis que le Muski, en lien avec l'hypertrophie de la capitale égyptienne, irrigue tout le territoire national (Bouhali, 2016). Comme l'écrit J.-C. David au sujet du Caire - mais cela fonctionne également pour Médina J'dida -, les facteurs expliquant la stabilité d'un quartier commerçant sont à chercher «dans les règles traditionnelles de fonctionnement du commerce, dans les pratiques des clientèles et des commerçants et leurs interactions, forces socio-économiques et proprement culturelles qui constituent le souk, suivant une fonctionnalité différente de celle des urbanistes ». Le poids du passé joue à plein : «la force des situations acquises (...) n'est pas seulement force d'inertie, mais valeur économique et foncière d'un site, fondée sur les habitudes des clientèles locales ou extérieures à la ville qui doivent se repérer facilement et trouver immédiatement le service dont elles ont besoin » (David, 1999, p. 236).

9 Ainsi, les circulations transnationales se sont greffées dans des quartiers commerciaux qui se sont structurés sur le temps long, et participent de leur requalification. De quartiers commerciaux plus ou moins délaissés dans la seconde moitié du $\mathrm{XX}^{\mathrm{e}}$ siècle, ils sont aujourd'hui des souks populaires pleinement intégrés à la scène des échanges globalisés, et constituent deux nœuds majeurs sur les routes du commerce transnational. 


\section{Réécriture du modèle du souk populaire par les produits de l'importation} d'importation made in China depuis la dernière décennie du $\mathrm{XX}^{\mathrm{e}}$ siècle. Ces produits ont sensiblement transformé les ambiances urbaines, en s'imposant sur les étals des boutiques et les tables des petits vendeurs de rue ${ }^{4}$, et en remplaçant bien souvent des marchandises auparavant produites sur place.

Cette diffusion et cette généralisation des articles d'importation ont en fait conservé les règles d'organisation qui président aux souks dans le monde arabe. Les marchés populaires sont en effet des marchés «découvert[s] et/ou couvert[s], ou bien un ensemble de rues (...) aux nombreuses petites boutiques regroupées en îlots spécialisés selon les marchandises vendues et/ou produites sur place (...) ou selon les professions exercées » (Desse et al., 2008). Les souks associent traditionnellement activités de vente, de production, de stockage des marchandises et d'accueil des populations marchandes étrangères à la ville. Au sein du souk, on trouve en général « des ruelles où sont regroupés les ateliers ou boutiques d'un même métier, et à l'extérieur, les différents marchés, notamment alimentaires, situés dans les quartiers ou les faubourgs" (Mermier et Peraldi, 2010). Cette organisation du marché par sous-ensembles spécialisés se retrouve aujourd'hui encore à Médina J'dida comme au Muski, comme le montre la carte du Muski ci-dessous (figure 2), entre spécialisation par type de produit et/ou spécialisation par type de vente (en gros ou au détail).

Figure 2. Spécialisation commerciale du Muski en 2013 : une répartition des produits conformes aux règles d'organisation d'un souk

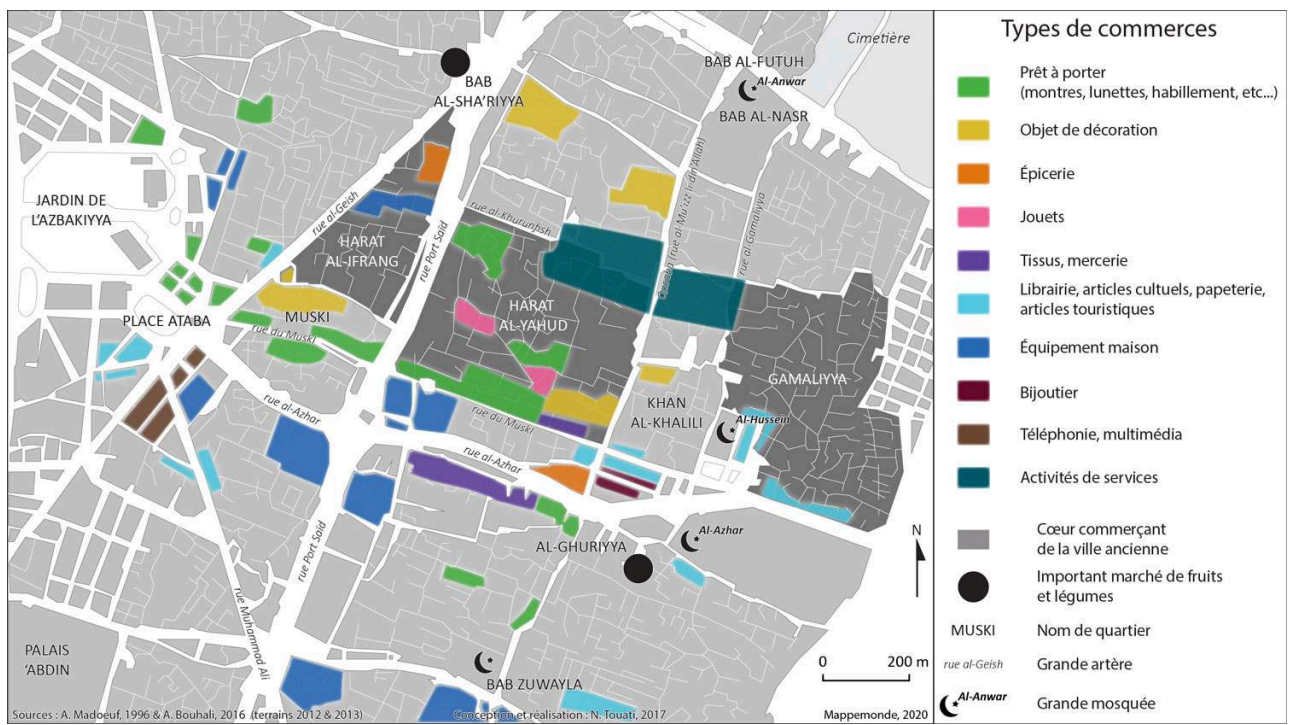

Donnés : Bouhali, 2016 ; cartographie : N. Touati, 2017

Néanmoins, on observe des changements dans le temps en ce qui concerne certaines rues ou certains sous-quartiers. Ces changements se sont opérés en quelques années seulement, ce que nous avons pu constater en comparant nos propres relevés de terrains à ceux réalisés antérieurement par d'autres chercheurs au Caire (Madœuf, 1997 ; David, 1999) comme à Oran (Semmoud, 1975 ; Mokrane, 2007). Des glissements 
discrets ont ainsi pu se dessiner. C'est le cas par exemple à Médina J'dida où le commerce de prêt-à-porter, bien représenté dans les années 1970, s'est renforcé tout au long des dernières décennies du XX $\mathrm{X}^{\mathrm{e}}$ siècle (figure 3). Les artisans-tailleurs qui étaient mentionnés dans les relevés de B. Semmoud ont disparu (carte 1972) et ont laissé la place à des grossistes spécialisés dans l'import et la vente de prêt-à-porter pour homme et enfant et de chaussures de sport. La vente de prêt-à-porter et de chaussures s'est largement étendue à Médina J'dida (cartes 2004 et 2014), devenant ainsi une des principales activités du quartier, et faisant petit à petit disparaître dans les ruelles à l'intérieur du quartier les grossistes spécialisés dans le tissu, et dans les avenues qui bordent le quartier ceux spécialisés dans les produits alimentaires.

Figure 3. Diffusion du commerce de prêt-à-porter et massification du made in China à Médina J'dida entre 1972 et 2014


Source des cartes : 1972 : Semmoud (1975) ; 2004 : Mokrane (2007) ; 2014 : Bouhali (2018)

Parfois, de véritables mutations ont lieu, impliquant un changement complet de la spécialisation commerciale d'une rue ou d'un îlot, l'ancienne spécialisation disparaissant presque complètement sous les assauts d'une nouvelle. C'est ce qui s'est passé par exemple au Muski, où le quartier anciennement spécialisé dans la papeterie et les produits d'emballage à destination des professionnels était en train de céder la place aux grossistes du prêt-à-porter, pour partie importé, pour partie fabriqué sur place dans des ateliers de confection à partir de tissus importés en gros d'Asie du Sudest $^{5}$. Les clichés de la figure 4 mettent en évidence cette transformation progressive du quartier, débutée il y a plus de dix ans. Les boutiques de vêtements ont commencé à s'y implanter alors que les papetiers le quittaient pour un quartier plus excentré, à la recherche de surfaces commerciales plus grandes, et pour bénéficier pour certains de l'aubaine que représente la vente d'une boutique ou d'un immeuble aux nouveaux acteurs du commerce transnational. L'arrivée de commerces spécialisés dans le prêt-àporter est liée à la présence de ces mêmes activités autour de la place Ataba, située juste derrière ces rues (figure 2) et qui est un des cœurs du prêt-à-porter. Autre facteur explicatif: l'existence d'entrepôts qui permettent le stockage de ces produits à quelques rues plus au nord dans le quartier, et l'approvisionnement régulier des boutiques à l'aide d'une flotte de porteurs à bras. En effet, l'entreposage des marchandises est un véritable enjeu dans un quartier où la place se fait rare et donc chère, et où les rues très étroites, en particulier dans le cas du Muski, et très densément fréquentées rendent difficile la circulation des marchandises. 
Figure 4. Au Muski, les grossistes en papier et carton laissent la place aux grossistes en prêt-àporter pour homme et à leurs centres commerciaux


Clichés : Bouhali, 2013

On remarque ainsi dans le nouveau quartier du commerce de prêt-à-porter en gros, la coexistence des deux spécialités: le papier et le vêtement, même si les surfaces commerciales spécialisées dans le vêtement en gros ont tendance à s'étendre au détriment du papier. Le commerce de papier en gros se maintient encore de façon résiduelle, principalement dans les boutiques les plus anciennes, et surtout dans celles qui sont relativement enclavées à l'intérieur du quartier, donc plus compliquées à approvisionner. Les grossistes en textile privilégient en effet des localisations plus visibles pour leurs boutiques, par exemple le long des rues les plus larges qui permettent de faciliter les approvisionnements, soit par charrette à bras, soit par véhicules triporteurs. Ces magasins se sont installés pour une grande partie d'entre eux dans des centres commerciaux récents, visibles à l'arrière-plan des clichés (figure 4).

Les rues des quartiers de Médina J'dida et du Muski deviennent ainsi des palimpsestes où les traces d'anciennes spécialisations se laissent deviner sous les nouveaux produits importés : une enseigne qui n'a pas été changée, quelques magasins ça-et-là qui sont les derniers à vendre un type d'article tandis que leurs voisins proposent tous des articles made in Asia. Face aux assauts des produits importés, rues et ruelles finissent par se ressembler, qu'on se trouve à Médina J'dida ou au Muski; les produits qu'on y vend sont finalement assez semblables, ce qui témoigne aussi de l'émergence et de l'affirmation d'un marché de consommation régional qui tend à s'uniformiser. Les produits made in China participent ainsi du renouvellement et de la réécriture du souk populaire arabe. 


\section{Renouvellement urbain en contexte mondialisé dans les souks du made in China}

16 Si l'activité commerciale est toujours concentrée dans les mêmes espaces urbains, si l'agencement des fonctions commerciales à l'intérieur de ces quartiers répond encore aux règles d'organisation du souk, le tissu commercial s'est adapté, les produits proposés ont changé, et, surtout, le bâti s'est renouvelé en profondeur. La morphologie assez souple de ces deux quartiers, héritée de plusieurs siècles d'histoire urbaine et bien souvent dans un mauvais état de conservation (figure 5), a offert des possibilités originales de reconversion et de transformation pour les activités du commerce transnational. Les formes anciennes du commerce ont été remodelées pour bénéficier du dynamisme économique lié à l'essor du commerce transnational, et de nouvelles formes urbaines apparaissent de façon concomitante dans ces quartiers de l'importation.

17 Le développement commercial récent a profité de la dégradation du tissu urbain de Médina J'dida et du Muski, dégradation dont les causes diffèrent selon le contexte urbain. À Oran, le statut très flou de la copropriété, en lien avec le peuplement des quartiers centraux lors de l'indépendance, en est une des causes. Les habitants qui sont venus remplacer les colons européens ont profité de biens vacants de l'État dont ils ont parfois pu devenir propriétaires dans les années 1980, mais sans qu'il y ait la mise en place de mécanismes de gestion de la copropriété (Bazin, 2013). Les immeubles n'ont ainsi pas ou peu été entretenus durant de longues années. En Égypte, les loyers des immeubles résidentiels anciens ont été bloqués sous la présidence de Nasser en raison de la pénurie de logements dans la capitale. Les loyers de certains immeubles sont ainsi restés à leur niveau des années 1950, d'où l'impossibilité pour les propriétaires d'entretenir les bâtisses. Cette situation a été aggravée au début des années 1990 par un tremblement de terre qui a secoué la capitale égyptienne le 12 octobre 1992, ce qui a de facto accéléré le processus de dégradation à l'œuvre et donné l'occasion aux propriétaires de détruire ces immeubles menaçant ruine et de les remplacer par des bâtisses plus adaptées aux activités commerciales alors en plein boom. 
Figure 5. Deux exemples d'immeubles dégradés parmi d'autres : à gauche à Médina J'dida et à droite au Muski
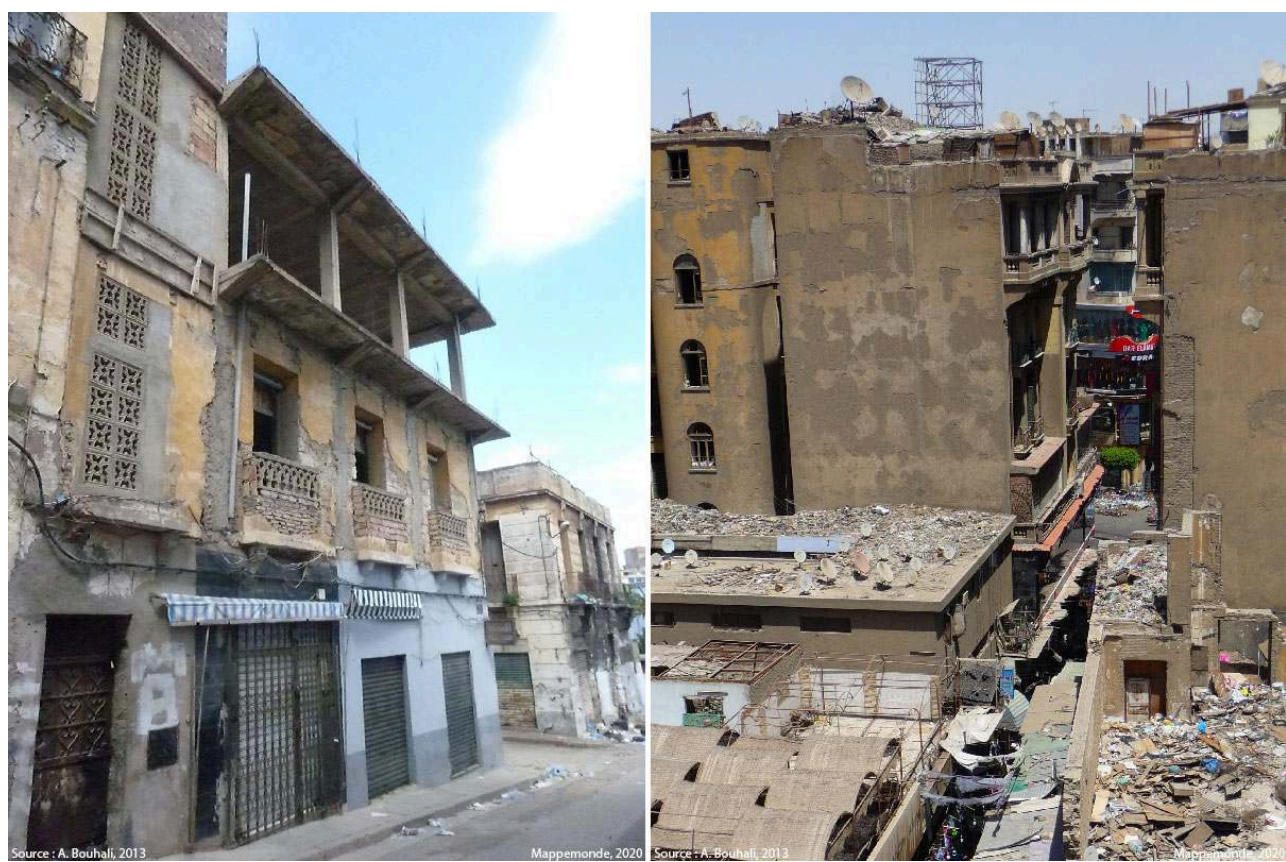

Clichés : Bouhali, 2013

Cette situation de dégradation et de flou a permis aux propriétaires immobiliers et aux commerçants de trouver un terrain à bâtir ou à reconstruire, dans un contexte de montée des prix de l'immobilier commercial et d'une demande croissante en locaux commerciaux, conséquences du succès de ces places marchandes. En effet, tout immeuble en état de dégradation est une possibilité nouvelle de développement immobilier et commercial dans un marché sous tension. Les paysages commerciaux, et en particulier le parc commercial, en sortent profondément transformés, à la fois par des opérations de réhabilitation et des opérations de rénovation urbaine ${ }^{6}$ menées à l'instigation de nombreux acteurs : propriétaires privés ayant bénéficié d'un héritage familial, ou entrepreneurs ayant dégagé suffisamment de bénéfices pour pouvoir investir dans la pierre.

Entrepreneurs-marchands et propriétaires ont ainsi pris de plus en plus de place dans la fabrique de la ville marchande en raison d'un contexte politique et institutionnel très favorable. Dans le cas cairote, ces propriétaires ont su profiter d'un moment de vacance du pouvoir et de moindre gestion de la ville par les autorités urbaines, en lien avec le printemps égyptien (Stadnicki, 2015). Dans le cas oranais, les propriétaires immobiliers ont su jouer de règles d'urbanisme assez vagues et d'un tuilage entre les différents acteurs impliqués dans la gestion et la planification de la ville pour obtenir des permis de construire dérogeant aux règles d'urbanisme. Dans les deux cas, les propriétaires ont su profiter de cette relative liberté pour construire de nouveaux immeubles commerciaux ou en détruire de plus anciens, sans respect des règlements en vigueur.

La modification des paysages commerciaux de ces quartiers s'est traduite tout d'abord par la rénovation d'une partie des façades des immeubles anciens et par l'aménagement de nouvelles vitrines (figure 6). La comparaison des façades des immeubles oranais et cairotes met en évidence des constantes dans le choix de la grammaire visuelle et des éléments de marketing urbain utilisés. Les couleurs vives sont fréquentes, en vue de se 
distinguer des autres boutiques. Les vitrines se généralisent, au rez-de-chaussée et de plus en plus dans les étages, au fur et à mesure que l'activité commerciale s'étend et investit les étages supérieurs des immeubles. La typographie utilisée pour le nom des magasins est moderne, et l'utilisation des caractères latins fréquente pour transcrire l'arabe. Le français dans le cas algérien et l'anglais dans le cas égyptien sont également très utilisés, deux langues qui signalent la mondialisation de la consommation? ${ }^{7}$.

Fig. 6. Au Muski (à gauche) et à Médina J'dida (à droite), les devantures rénovées des locaux commerciaux contrastent fortement avec le bâti existant



Clichés : Bouhali, 2013 et 2014

21 À côté de la réhabilitation des façades, un processus de rénovation urbaine a conduit à la destruction d'immeubles et d'îlots anciens et à la construction de centres commerciaux à l'initiative de propriétaires immobiliers du quartier, parfois eux-mêmes commerçants et qui souhaitaient profiter de la rente foncière et immobilière du quartier. Ce second type d'opération urbaine entraîne parfois un remodelage spectaculaire des paysages urbains, et participe de façon évidente à la modernisation architecturale et visuelle de ces quartiers, un indicateur de la circulation de modèles urbains mondialisés qui accompagne celle des produits (figure 7) (Bouhali, 2018a). Le style architectural de ces nouveaux immeubles commerciaux tranche avec le style des bâtiments plus anciens. L'utilisation de matériaux modernes pour les façades est fréquente (verre teinté, enduits clinquants), ce qui jure avec l'uniformité des quartiers commerciaux. On remarque également que la verticalité de ces immeubles, qui permet d'accroître les surfaces commerciales en vue de répondre à la très forte demande en locaux commerciaux, est aussi une façon pour les promoteurs de souscrire aux codes de la grammaire architecturale mondiale. C'est ce que nous a dit en substance un commerçant qui avait initié un projet de construction d'une tour commerciale en plein cœur de la ville ancienne du Caire. Cet homme s'était inspiré des tours et des centres commerciaux modernes et globalisés que l'on trouve dans les quartiers d'affaires du Grand Caire, principalement en périphérie ou dans le centre-ville moderne (Abaza, 2006). Le mall trouve ainsi sa place dans les quartiers populaires du made in China, montrant que les chalands et les commerçants arabes "partagent l'idée que les shopping malls incarnent la manière moderne de consommer » (Cohen, 1996: 1062, cité par Assaf et Camelin, 2017).

22 Les paysages commerciaux obtenus sont extrêmement variés: dans une impasse commerciale du Muski ou dans une ruelle de Médina J'dida, on peut trouver côte à côte un immeuble vétuste ou menaçant ruine, un immeuble ancien sur lequel aura été plaquée une nouvelle devanture "bling bling» et un centre commercial moderne 
flambant neuf (figure $\mathbf{7 a}$ et $\mathbf{7 b}$ ). Le bâti devient de plus en plus hétéroclite dans ces deux centralités marchandes, et est marqué par l'influence des villes mondiales, non pas tant occidentales, mais plutôt celles fréquentées par les commerçants égyptiens et algériens sur les routes du commerce transnational : Dubaï, Abu Dhabi, et les grandes villes asiatiques (Bouhali, 2018a).

Figure 7a. Centres commerciaux récents ou en construction au Muski

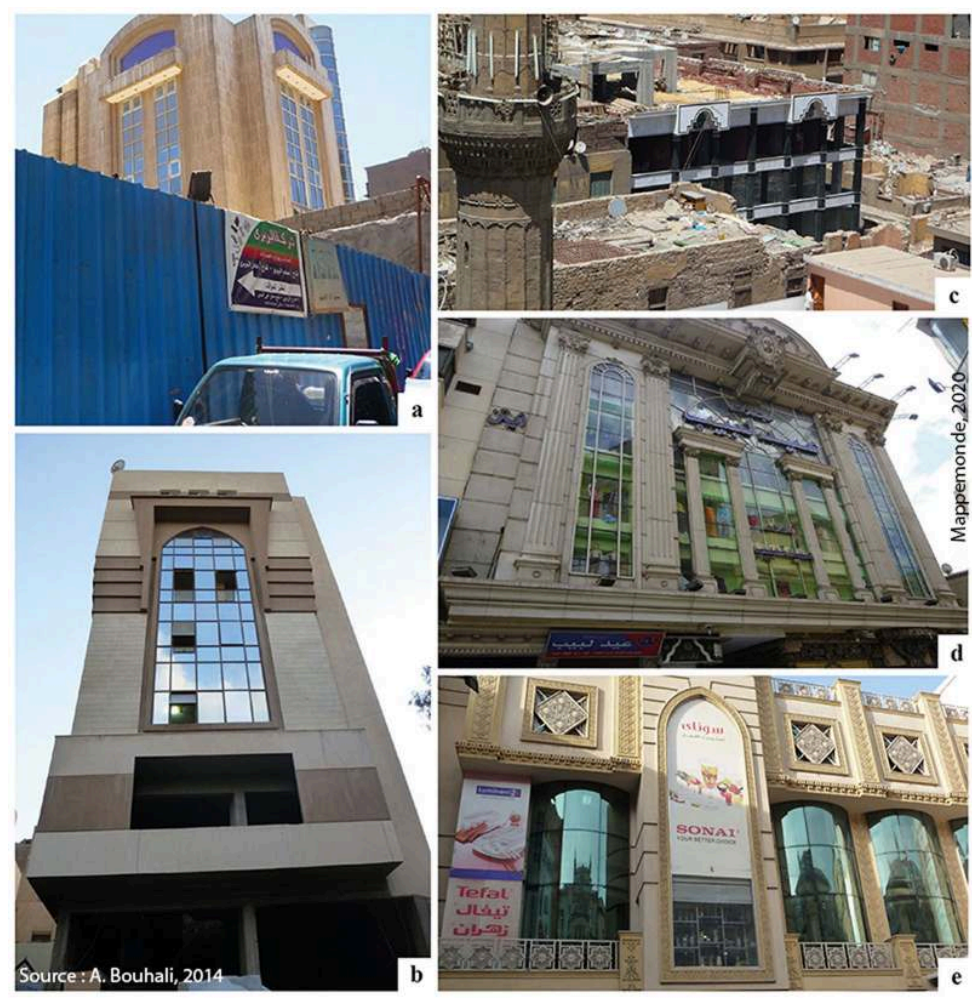

Clichés : Bouhali, 2011-2014 
Figure $7 \mathrm{~b}$. Centres commerciaux récents ou en construction à Médina J'dida



Clichés : Bouhali, 2011-2014

23 Ce vaste mouvement de renouvellement urbain et d'hyper concentration commerciale est venu amplifier un processus déjà existant dans ces deux quartiers : la diminution de la population résidente. Ce processus s'inscrit dans une tendance actuelle qui va dans le sens de la dynamique générale des centres-villes plus largement, au nord (Van Criekingen et Fleury, 2006; Zukin et al., 2009; Gonzalez et Waley, 2013 ; Cócola Gant, 2015) comme au sud: celle de la tertiarisation des centres urbains, de leur gentrification par le développement d'activités commerciales, et de la déconcentration de leur population en direction des périphéries. À Médina J'dida et au Muski, le déclin de la fonction résidentielle est semble-t-il inversement proportionnel à la densification commerciale des rues et ruelles de ces deux quartiers-marchés (figure 8 et figure 9). 
Figure 8. Évolution de la population de la vieille ville du Caire entre 1986 et 2006

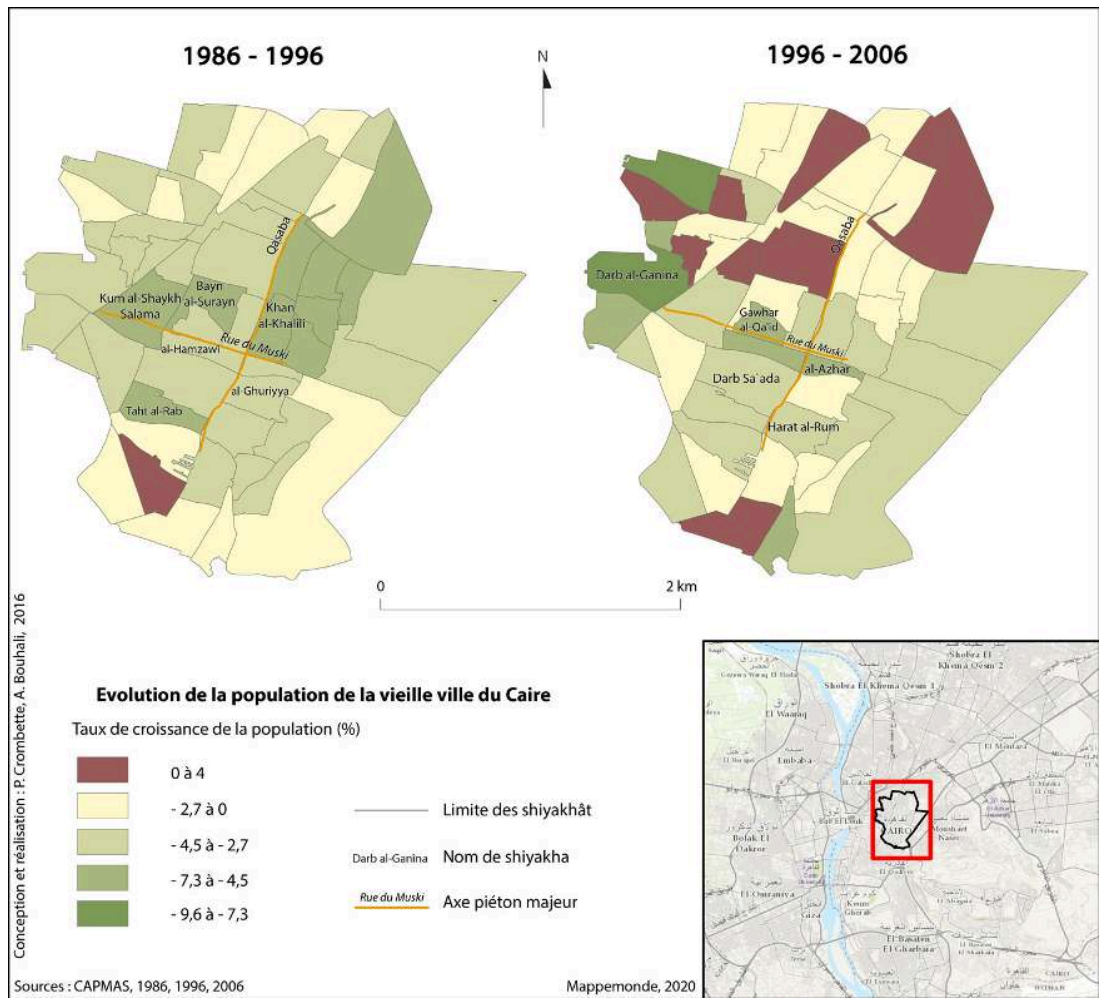

Réalisation : P. Crombette, 2016. Données : Capmas

Figure 9. Médina J'dida

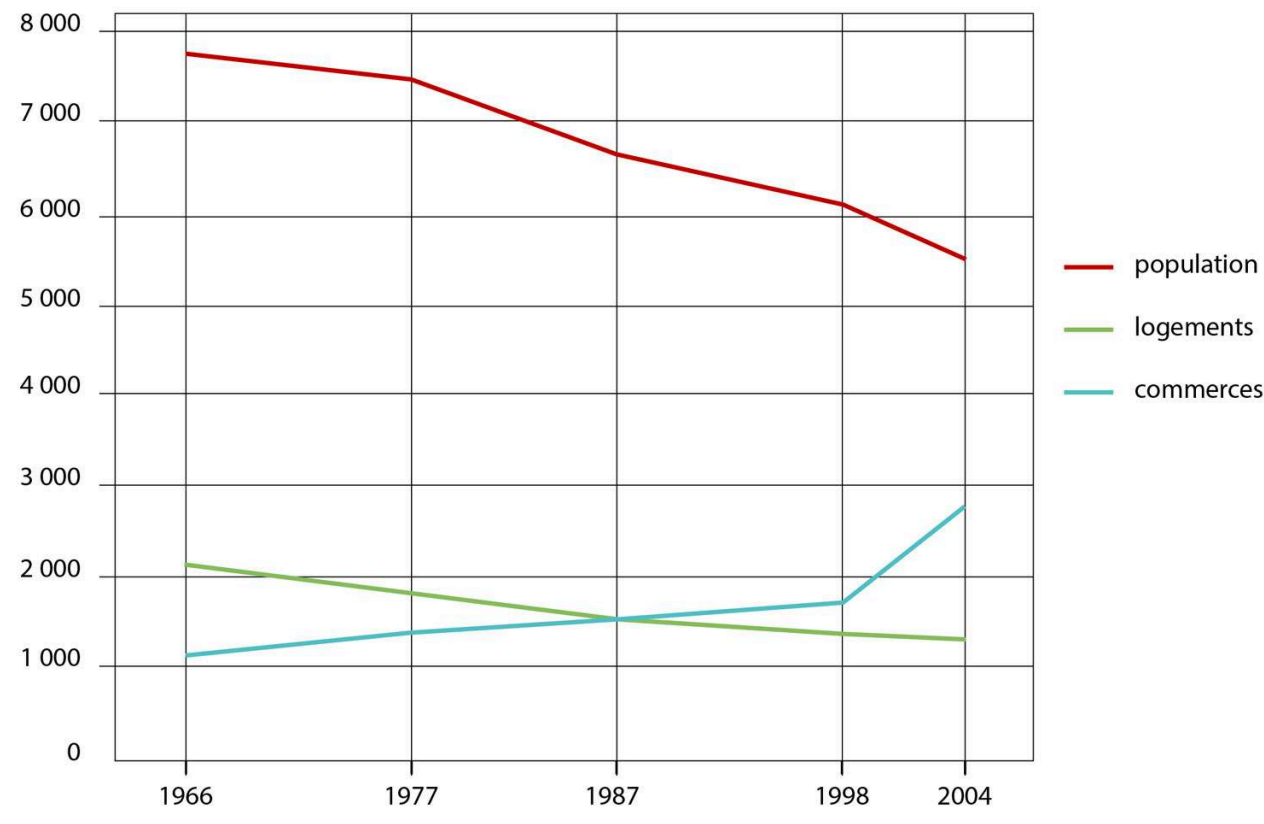

Sources : En quête de terrain - ONS

Réalisation : S. Mokrane, 2007

Mappemonde, 2020

Évolution comparée de la population, du parc de logements et des commerces.

Source : Mokrane, 2007, p. 108 
Cette baisse de la fonction résidentielle au profit des activités commerciales s'exprime de différentes manières: les temporalités et les rythmes des quartiers reposent désormais largement sur les temporalités des activités commerciales; les commerces de proximité sont inexistants et/ou disparaissent au profit de services à destination d'une clientèle exogène ; la population habitante est repoussée dans les périphéries du quartier. Cette rivalité entre fonction résidentielle et fonction commerciale ne se fait pas sans heurt. Des conflits éclatent parfois, entre les commerçants, les propriétaires qui souhaitent voir se développer le commerce et donc la rente immobilière, et les habitants qui refusent de quitter ces quartiers de leur plein gré. Un tel conflit nous avait été par exemple rapporté au Muski par des commerçants installés dans un des nouveaux quartiers en plein boom de la vaisselle et de l'électroménager en juin 2013. Un propriétaire avait volontairement causé des dégradations à son immeuble afin de pouvoir en expulser les locataires qui bénéficiaient de loyers bloqués, nécessitant l'intervention de la police locale. Lors de nos enquêtes, l'immeuble avait été vidé de ses habitants, et était en attente de travaux.

Le développement du commerce transnational est ainsi un facteur de recomposition profonde de ces quartiers commerçants, à l'origine non seulement d'un renouvellement urbain qui diffuse un urbanisme commercial de plus en plus uniformisé et mondialisé, mais également de transformations économiques et sociales, avec la baisse de la fonction résidentielle face à la pression commerciale. Ces quartiers du commerce transnational sont produits et diffusés par les marchands, les propriétaires et les investisseurs, qui opèrent par le bas, devenant ainsi des acteurs majeurs de la fabrique de la ville.

\section{Conclusion}

Un modèle du quartier commerçant du made in China s'est ainsi développé et diffusé le long des routes du commerce transnational. Les études de cas montrent ainsi qu'une forme d'urbanisme commercial uniformisé et mondialisé s'est constituée par le bas, à travers l'importation de produits à destination d'un marché de consommation régional, à travers l'homogénéisation du bâti commercial, et surtout l'introduction et le développement des centres commerciaux à destination des catégories populaires et moyennes. Le Muski et Médina J'dida ne sont que deux exemples parmi d'autres de ce type de quartier. À titre de comparaison, on citera notamment le marché Bou Mendil dans la médina de Tunis (Doron, 2015), Choupot et les Castors à Oran (Boudinar et Belguidoum, 2015), le marché Capitale à Nouakchott (Mauritanie) ainsi que Derb Omar et Derb Sultan à Casablanca (Maroc), trois marchés dans lesquels nous avons débuté des enquêtes en 2017 et $2018^{8}$. Médina J'dida, le Muski et tous les autres sont ainsi révélateurs à la fois de la circulation des produits, mais aussi de celle des modèles de consommation et des modèles urbains en Afrique du Nord, en direction des consommateurs les plus modestes.

Les deux quartiers étudiés ici soulignent également le rôle des commerçants et des propriétaires fonciers et immobiliers dans la construction et la diffusion de ce modèle, des acteurs qui sont aujourd'hui des forces vives dans la fabrique de ces marchés et de la ville plus largement. En ce sens, ils participent à leur échelle de la fabrique de la ville mondialisée côté sud (Robinson, 2002), en étant à l'origine d'un processus d'uniformisation de la ville (Smith, 1982) tant au Maghreb qu'au Machrek. 
Enfin, les transformations récentes qui touchent ces quartiers et leur spécialisation croissante dans le commerce transnational permettent de participer aux débats concernant la gentrification commerciale (Bridge et Dowling, 2001; Smith, 2005 ; Van Criekingen et Fleury, 2006; Zukin et al., 2009), côté sud cette fois-ci. Face au développement des activités du commerce transnational, rente immobilière et rente foncière ne cessent d'augmenter, entraînant progressivement le départ des habitants les plus pauvres. Cet article permet ainsi de mettre en évidence le rôle des activités commerciales menées par des acteurs discrets dans les processus de gentrification.

\section{BIBLIOGRAPHIE}

ABAZA M. (2006). Changing consumer cultures of modern Egypt. Cairo's urban reshaping. Leyde : Brill, 309 p. ISBN 90-04-15277-6

ABU-LUGHOD J. (1965). "Tale of two cities: the origins of Modern Cairo". Comparative Studies in Society and History, vol. 7, $\mathrm{n}^{\circ}$ 4, p. 429-457.

ASSAF L., CAMELIN S. (2017). « Shopping malls : l'avènement de la modernité ? Introduction au numéro ». Ateliers d'anthropologie, $\mathrm{n}^{\circ}$ 44. En ligne : https://journals.openedition.org/ateliers/ 10365

BAZIN L. (2013). «L'État endetté en Algérie. Demande d'État, conflits sociaux et ressorts imaginaires du pouvoir ». In HOURS B., OULD-AHMED P. (dir.), Dette de qui, dette de quoi ? Une économique anthropologique de la dette. Paris : L'Harmattan, p. 171-200. ISBN 978-2-343-02074-7

BEKKOUCHE A. (2005). « Un quartier nommé ville nouvelle. Du village nègre colonial à la Médina J'dida ». Annales de la recherche urbaine, $\mathrm{n}^{\circ} 98$.

BELGUidoum S., CHOUADRA S., ALIKHOdJA N. (2017). «El Eulma (Algérie), anatomie d'une place emblématique du commerce transnational ». En ligne : https://hal.archives-ouvertes.fr/ halshs-01508982

BELGUIDOUM S., PLIEZ O. (2012). « Construire une route de la soie entre l'Algérie et la Chine ». Diasporas, $\mathrm{n}^{\circ} 20$, p. $115-130$.

BELGUIDOUM S., PLIEZ O. (2015). « Made in China. Commerce transnational et espaces urbains autour de la Méditerranée ». Les Cahiers d'EMAM. Études sur le Monde Arabe et la Méditerranée, n 26 . En ligne : https://journals.openedition.org/emam/909

BERTONCELLO B., FETTAH S. (2007). «Introduction. Centralités en mouvement ». Rives méditerranéennes, $\mathrm{n}^{\circ}$ 26, p. 5-8. En ligne : https://journals.openedition.org/rives/791

BOUDINAR A., BELGUIDOUM S. (2015). « Dynamique marchande et renouveau urbain à Oran. Médina J'dida et Choupot, deux quartiers du commerce transnational ». Les Cahiers d'EMAM. Études sur le Monde Arabe et la Méditerranée, $n^{\circ}$ 26. En ligne : https://journals.openedition.org/emam/1027 BOUHALI (2015). « Médina J'dida (Oran), un quartier-marché sur les routes algériennes du commerce transnational ». Les Cahiers d'EMAM. Études sur le Monde Arabe et la Méditerranée, $\mathrm{n}^{\circ} 26$. En ligne : https://journals.openedition.org/emam/889 
BOUHALI (2016). « Ancien lieu du commerce et nouveau commerce transnational du made in China au marché al-Muski (Le Caire) : une fabrique de la ville par le bas sous tensions ». In DESSE R.-P., LESTRADE S. (dir.), Mutations de l'espace marchand. Rennes : Presses Universitaires de Rennes, p. 353-369. ISBN 978-2-7535-4899-2

BOUHALI (2018a). « Les places marchandes du made in China au Caire et à Oran : mondialisation et transformations des espaces et des pratiques de consommation ». Bulletin de l'association de géographes français, $\mathrm{n}^{\circ}$ 95-2, p. 222-238.

BOUHALI (2018b). "Negotiating Streets and Space in Transnational Trade Marketplaces in Oran (Algeria) and Cairo (Egypt): 'Place Struggle' in the Commercial City”. Articulo - Journal of Urban Research, $\mathrm{n}^{\circ}$ 17-18. En ligne : https://journals.openedition.org/articulo/3369

BRIDGE G., DOWLING R. (2001). “Microgeographies of Retailing and Gentrification”. Australian Geographer, vol. 32, nº 1, p. 93-107.

CHOPLIN A., PLIEZ O. (2015). “The inconspicuous spaces of globalization”. Articulo - Journal of Urban Research, $\mathrm{n}^{\circ} 12$. En ligne : https://journals.openedition.org/articulo/2905

CHOPLIN A., PLIEZ O. (2018). La mondialisation des pauvres. Loin de Wall Street et de Davos. Paris, France : Seuil, 108 p. ISBN 978-2-02-136652-5

Cócola GANT A. (2015). “Tourism and commercial gentrification". Communication présentée à la conférence internationale RC21 "The Ideal City: between myth and reality. Representations, policies, contradictions and challenges for tomorrow's urban life”, Urbino, Italie.

DARBON D. (2012). «Classe(s) moyenne(s) : une revue de la littérature ». Afrique contemporaine $\mathrm{n}^{\circ}$ 244, p. 33-51.

DAVID J.-C. (1999). «Centralités anciennes et actuelles dans “Al-Qahira” ». In DENOIX S., DEPAULE J.-C., ARNAUD J.-L., TUCHSCHERER M. (dir.), Le Khan al-Khalili et ses environs : un centre commercial et artisanal au Caire du XIII ${ }^{e}$ au XX ${ }^{e}$ siècle. Le Caire : Institut français d'archéologie orientale, p. 209-244. ISBN 978-2-7247-0211-8

DE KONING A. (2009). Global Dreams. Class, gender, and public space in Cosmopolitan Cairo. Le Caire/NewYork : The American University in Cairo Press, 195 p. ISBN 9774162498

DESSE R.-P. et al. (dir.). (2008). Dictionnaire du commerce et de l'aménagement. Rennes : Presses Universitaires de Rennes, 357 p. ISBN 978-2-7535-0684-8

DORON A. (2015). “Bou Mendil Market, Tunis”. In P. Mörtenböck, H. Mooshammer (dir.), Informal market worlds atlas: the architecture of economic pressure, Rotterdam : Nai010 Publishers, p. 224-229.

Encyclopédie de l'Islam. Tome IX, San-Sze. (1998). Leyde, Pays-Bas : E.J. Brill.

GONZALEZ S., WALEY P. (2013). “Traditional Retail Markets: The New Gentrification Frontier?”. Antipode, vol. 45, n 4, p. 965-983.

ILBERT R. (1982). « Le Caire a-t-il une médina ?». Fascicule de Recherches URBAMA, $\mathrm{n}^{\circ}$ 10-11, p. 263-281.

MADCUF A. (1997). Images et pratiques de la ville ancienne du Caire : les sens de la ville. Tours : Université François-Rabelais, thèse de doctorat en géographie, $570 \mathrm{p}$.

MATHEWS G., ALBA VEGA C. (2012). “Introduction. What is globalization from below?”. In G. MATHEWS, G. L. RIBEIRO, C. ALBA VEGA (dir.), Globalization from below: the world's other economy, Londres :

Routledge, p. 1-15. ISBN 978-0-415-53508-3 
MERLIN P., CHOAY F. (2015) Dictionnaire de l'urbanisme et de l'aménagement. Paris : Presses universitaires de France, $4^{\text {ème }}$ éd., 1024 p. ISBN 978-2-13-063068-5

MERMIER F., PERALDI M. (dir.). (2010). Mondes et places du marché en Méditerranée. Formes sociales et spatiales de l'échange. Paris : Karthala, 337 p. ISBN 978-2-8111-0416-0

MEYER G. (1988). "Manufacturing in old quarters of Central Cairo". Fascicule de Recherches URBAMA, $\mathrm{n}^{\circ} 19$, p. $75-90$.

Mokrane S. (2007). Évolution de la structure commerciale dans un quartier ancien d'Oran : Médina J'dida. Oran : Faculté des Sciences de la Terre, de Géographie et d'Aménagement du territoire, mémoire de magister non publié.

PLIEZ O. (2007). « Des jeans chinois dans les rues du Caire, ou les espaces discrets de la mondialisation ». Mappemonde, $\mathrm{n}^{\circ}$ 88. En ligne : https://mappemonde-archive.mgm.fr/num16/ articles/art07404.html

RAYMOND A. (1993). Le Caire. Paris : Fayard, 428 p. ISBN 2-213-02983-0

ROBINSON J. (2002). "Global and world cities. A view from off the map". International Journal of Urban and Regional Research, vol. 26, $\mathrm{n}^{\circ} 3$, p. 531-554.

SEMMOUD B. (1975). Médina Jdida : étude cartographique et géographique d'un quartier d'Oran. Paris : Université Paris Diderot, thèse de $3^{\mathrm{e}}$ cycle, $169 \mathrm{p}$.

SMITH N. (1982). “Gentrification and Uneven Development”. Economic Geography, vol. 58, n² 2, p. 139-155.

SMITH N. (2005). The New Urban Frontier : Gentrification and the Revanchist City. Londres : Routledge, 288 p. ISBN 978-0-203-97564-0

SOUMAGNE J. (2008). « Paysage commercial ». In DESSE R.-P. et al., (dir.) Dictionnaire du commerce et de l'aménagement. Rennes : Presses Universitaires de Rennes.

STADNICKI R. (2015). « Dynamiques urbaines en Égypte depuis la révolution de 2011 : ruptures et continuités ». In Rougier B., Lacroix S. (dir.), L’Égypte en révolution(s). Paris : Presses Universitaires de France, p. 271-290.

VAN CRIEKINGEN M., FLEURY A. (2006). « La ville branchée : gentrification et dynamiques commerciales à Bruxelles et à Paris ». Belgeo. Revue belge de géographie, $\mathrm{n}^{\circ}$ 1-2, p. 113-134. En ligne : ZUKIN S. et al. (2009). "New Retail Capital and Neighborhood Change: Boutiques and Gentrification in New York City". City \& Community, vol. 8, $n^{\circ} 1$, p. 47-64.

\section{NOTES}

1. Le terme de souk est passé dans la langue française pour désigner les marchés populaires dans le monde arabe. Ce terme désigne à la fois «l'échange commercial de biens ou de services et le lieu dans lequel se déroule cet échange » (Encyclopédie de l'Islam, 1998).

2. Il est impossible de chiffrer précisément les volumes de biens de consommation courante importés, ou même leur valeur. Une partie de ces marchandises passe en effet sous les outils de mesures statistiques des gouvernements algérien et égyptien, car les entrepreneurs qui sont à l'origine de ces importations sous-estiment auprès des douanes les valeurs des marchandises qu'ils achètent en Asie afin d'échapper aux taxes. 
3. Par paysages commerciaux, nous entendons à la fois le parc commercial, c'est-à-dire les magasins et leur assemblage linéaire (rues, passages, corridors), les enseignes et la publicité, ainsi que la voirie vouée à la desserte du commerce et au lèche-vitrines (Soumagne, 2008).

4. La présence des vendeurs de rue s'est largement étoffée entre 2011 et 2014 dans les rues des quartiers étudiés, en raison de la moindre présence des autorités urbaines et de la police municipale dans le contexte politique et social troublé des printemps arabes. Ce point fait l'objet d'une autre publication (Bouhali, 2018b).

5. Ni l'Égypte ni l'Algérie ne sont en mesure de fournir le textile nécessaire à la confection pour répondre à la demande, et les ateliers et/ou les fabriques qui se sont maintenus ou qui se sont développés dans les grandes villes utilisent essentiellement des matières premières importées de l'étranger, notamment de Chine.

6. La réhabilitation désigne des " procédures visant la remise en état d'un patrimoine architectural et urbain longtemps déconsidéré et ayant récemment fait l'objet d'une revalorisation économique, pratique et/ou esthétique (...). [Cela] suppose un respect du caractère architectural du bâtiment ». La rénovation urbaine quant à elle implique " la démolition en vue d'une construction nouvelle, d'un secteur urbain », terme impropre donc pour désigner un processus de démolition-reconstruction (Merlin et Choay, 2015).

7. L'anglais est en Égypte un héritage de la période coloniale britannique. Il était jusqu'à récemment plutôt un signe de l'internationalisation des élites culturelles et financières égyptiennes (de Koning, 2009). Sa diffusion auprès des classes populaires et des classes moyennes est selon nous un indice supplémentaire de leur participation à la mondialisation à travers notamment la consommation de produits importés.

8. Ces enquêtes ont bénéficié du soutien financier du LABEX DynamiTe et du Laboratoire Mixte International MOVIDA en 2017 et 2018.

\section{RÉSUMÉS}

À partir de l'étude de deux quartiers-marchés spécialisés depuis la fin des années 1990 dans les produits de consommation courante fabriqués en Chine à destination des classes moyennes et populaires urbaines, Médina J'dida à Oran (Algérie) et le Muski au Caire (Égypte), cet article montre que, face au déploiement des routes de l'échange entre Asie et monde arabe, on assiste à la fabrique d'un modèle de quartier de l'importation dans la région. À la mondialisation des échanges et des mobilités de marchandises et de personnes répond ainsi une banalisation des formes urbaines du commerce du made in China.

This paper compares the urban development of two marketplaces specialised in retail and wholesale trade of made-in-China goods since the 1990s: Medina J'dida in Oran (Algeria) and the Muski in Cairo (Egypt). In response to the expansion of trade routes between Asia and the Arab world, a new, import district model is under development in the region. The globalisation of commodity flows and human mobilities thus results in a standardisation of urban forms in transnational trade districts.

A partir del estudio de dos barrios -Médina J'dida en Orán (Argelia) y Muski En El Cairo (Egipto)especializados desde finales de la década de 1990 en el comercio de productos fabricados en China para la clase media urbana y trabajadora, se analiza la reconfiguración de los espacios 
comerciales tradicionales en otros especializados en la importación de mercanías asiáticas. Sus transformaciones están produciendo una homogeneización, trivialización y degradación del tejido urbano. Estos cambios solo se explican por la creciente movilidad e intercambios de mercancías y de personas a través de las rutas comerciales entre China y los países árabes.

\section{INDEX}

Thèmes : Les routes de la soie existent déjà. Routes transnationales et places marchandes du made in China entre Asie Afrique et Europe

Palabras claves : comercio transnacional, tejido urbano, mercados, modelo urbano, globalización

Mots-clés : commerce transnational, fabrique de la ville, marchés, modèle urbain, mondialisation

Keywords : globalisation, making of the city, markets, transnational trade, urban model

\section{AUTEUR}

\section{ANNE BOUHALI}

Géographe, MCF à l'Université de Picardie, équipe d'accueil « Habiter le monde » EA 4287 et chercheuse associée à l'UMR 8586 PRODIG 